\title{
Toxin Inactivation in Toxin/Antitoxin Systems
}

\section{Laura Fernandez-Garcia ${ }^{1,3}$, Jun-Seob Kim ${ }^{4}$, Maria Tomas ${ }^{3}$, and Thomas K. Wood ${ }^{1,2, *}$}

${ }^{1}$ Department of Chemical Engineering and ${ }^{2}$ Department of Biochemistry and Molecular Biology,

Pennsylvania State University, University Park, Pennsylvania, 16802-4400, USA

${ }^{3}$ Microbiology Department-Research Institute Biomedical A Coruña (INIBIC), Hospital

A Coruña (CHUAC), University of A Coruña (UDC), A Coruña, Spain

${ }^{4}$ Infectious Disease Research Center, Korea Research Institute of Bioscience \& Biotechnology (KRIBB) 125 Gwahak-ro, Yuseong-gu, Daejeon 34141, South Korea

*For correspondence. E-mail tuw14@psu.edu

Tel.(+)1 814-863-4811; Fax (1) 814-865-7846

Running title: Promoter mutations inactivate toxins.

Keywords: toxin, antitoxin, mutation

Key Contributions: In this manuscript we determined that (i) toxins may be inactivated in 6 to 12 hours due to mutations, (ii) these mutations occur primarily in the promoters of the toxins and not in the structural genes, and (iii) chromosomal proteins IraM and MhpR are necessary for toxin activity. 


\begin{abstract}
A BSTRACT
Toxin/antitoxin (TA) systems are used primarily to inhibit phage, reduce metabolic activity during stress, and maintain genetic elements. Given the extreme toxicity of some of the toxins of these TA systems, we were curious how the cell silences toxins, if the antitoxin is inactivated or when toxins are obtained without antitoxins via horizontal gene transfer. Here we find that the RalR (type I), MqsR (type II), GhoT (type V), and Hha (type VII) toxins are inactivated primarily by a mutation that inactivates the toxin promoter or via the chromosomal mutations iraM and $m h p R$.
\end{abstract}

\title{
INTRODUCTION
}

Toxin-antitoxin (TA) systems have been related to gene regulation during the stress response [1], persister cell generation [2,3], bacteriophage protection [4], and other functions [5]. TA systems were originally found on plasmids [6], but they have also been found in bacterial chromosomes and bacteriophages. Almost all bacteria have TA systems in their genomes, reaching 88 TA loci in case of Mycobacterium tuberculosis; hence, TA systems are related to pathogenicity [7]. Furthermore, TA systems have also been described in 86 Archaea and even in some fungi, in which there have been identified fourteen Doc toxin homologs [8].

TA systems are primarily two component systems, composed of a toxin, which disrupts important cellular mechanisms, and an antitoxin, which blocks the toxin action [5]. Depending on how the antitoxin interacts with the toxin, TA systems are classified into seven types. In type I systems (e.g., Hok/Sok), the antitoxin is an antisense RNA of the toxin. In type II (e.g., CcdB/CcdA) and III (e.g., ToxN/ToxI) systems, the antitoxin protein or RNA, respectively, inhibits the toxin by direct binding [5]. In type IV systems (e.g., $\mathrm{CbtA} / \mathrm{CbeA}$ ), the antitoxin competes with the toxin for the target [9], and in type $\mathrm{V}$ systems (e.g., GhoT/GhoS), the antitoxin is an enzyme that cleaves specifically the toxin mRNA [10]. In type VI systems (e.g., SocB/SocA), the antitoxin protein facilitates toxin degradation as an adaptor protein [7]. Recently, the type VII system has been described (Hha/TomB) in which the antitoxin is an enzyme that inactivates the toxin by oxidizing a cysteine residue $[11,12]$. 
The broad distribution and myriad types of TA systems implies there is an evolutionary advantage for utilizing them. Nevertheless, TA systems require tight regulation, to prevent undesired reductions in metabolism. For example, deletion of the gene encoding antitoxin MqsA is lethal [13]. For this tight regulation, TA systems are often self-regulated, as (i) antitoxins of type II TAs repress expression via promoter binding, (ii) some toxins limit both antitoxin and toxin levels via post-transcriptional cleavage of mRNA [14,15], and (iii) some antitoxins inhibit toxins. For example, toxin MqsR autoregulates itself by cleaving its own mRNA [16].

This study focuses on four different toxins, MqsR, GhoT, RalR, and Hha, each one from a different TA system. The MqsR/MqsA system is a type II TA system in which the toxin, MqsR, was originally characterized as a biofilm formation regulator that is quorum-sensing related [17]. MqsR also regulates another TA system, GhoT/GhoS [18]. MqsA antitoxin participates in the global stress response by regulating RpoS and activating biofilm formation under oxidative stress [1]. As an example of this oxidative stress response, the MqsR/MqsA system manages growth during stress due to bile acids in the gastrointestinal tract [19]. MqsR is also the first toxin that when inactivated, reduces persister cell formation [20]. In regard to its regulation, toxin MqsR disrupts the MqsA-DNA complex to activate transcription [16].

The first type V TA system, GhoT/GhoS [10], is named based on the ghost-cell phenotype seen when toxin GhoT is produced, which results from membrane damage and reduces ATP [21]. The RalR/RalA system is a type I TA system found in the cryptic prophage rac [22]. RalR is the only known, non-specific DNase TA system toxin (many toxins are RNases); it activates the SOS response to DNA damage and increases resistance to the antibiotic fosfomycin. Hence, the RalR/RalA systems improve bacterial fitness under stress conditions [22].

Three years ago, the first type VII TA system was described [11]. The haemolysin expression modulation protein (Hha) is the toxin, and TomB is the antitoxin. Hha is a global transcriptional regulator that modulates cell physiology [23] by (i) forming the Hha-H-NS complex where it represses the pathogenicity locus of enterocyte effacement (LEE), (ii) repressing the transcription of rare codon tRNAs (bacteriolytic effect) and fimbrial genes which reduces biofilm formation [24], (iii) having a pleiotropic 
effect in catabolite repression [25], inducing protease ClpXP, which activates some prophage lytic genes [24], and inducing excision of prophages Cp4-57 and DLP-12 of E. coli [26]. Remarkably, instead of forming a complex between the toxin and the antitoxin, toxin Hha is inactivated by oxidation mediated by the antitoxin TomB [11].

The aim of this study was to provide insights into how bacteria cope with TA toxin activity by identifying what mutations take place in bacteria to inactivate toxins, for cases in which antitoxins do not function or are not present, such as after horizontal gene transfer. For this goal, we produced each toxin (an RNase, membrane-damaging peptide, DNase, and transcriptional regulator) from a common plasmid backbone and promoter, and we determined that bacteria inactivate toxins primarily by mutating the promoter of the toxin or by mutating chromosomal copies of the iraM and $m h p R$ genes.

\section{RESULTS}

Mutation analysis. To determine general insights into how toxins are inactivated, we studied four diverse toxins by choosing one from each of the type I (RalR), type II (MqsR), type V (GhoT) and type VII (Hha) systems. Initially, each toxin inhibited cell growth completely when induced with $1 \mathrm{mM}$ IPTG, and each antitoxin was able to mask each toxin, restoring growth to that seen with the empty plasmid for antitoxins MqsA, RalA, and TomB whereas GhoS did not completely restore growth (Fig. 1).

For all four toxins, after 6 to $12 \mathrm{~h}$, growth inhibition ceased (Fig. 1) which indicated a mutation in the plasmid or in the chromosome that allowed the cells to resume growth. Also, purified colonies, obtained after the toxicity of each of the four toxins was inactivated, grew in the presence of IPTG (Fig. 2), indicating the mutations were stable.

Upon sequencing the plasmids of the strains with inactivated toxins, eight plasmids (two encoding MqsR, four encoding GhoT, and two encoding RalR), showed a partial deletion of a core 32 nucleotides in the Pt5-lac promotor rather than changes in the structural portion of the gene (Fig. S1-S4). Specifically, the core deletion included the -10 TATA box of the promotor. For 10 other strains with inactivated toxins (one MqsR, one GhoT, three RalR, and five Hha), no mutation in the promoter and toxin gene were found (Fig. 
S1-S4); hence, a stable mutation occurred in the chromosome. Note that along with the five mutants with chromosomal changes that inactivate Hha shown in Fig. 2, another three mutants were obtained with chromosomal changes that inactivate Hha, so in all, Hha was inactivated eight times by changes in the chromosome.

To investigate changes in the chromosome that led to growth in the presence of the four toxins in the absence of changes in the promoter and toxin genes, we selected five strains (one for MqsR, one for GhoT, two for RalR and one for Hha) and sequenced their chromosomes. We found several single nucleotide changes, including those in bacteriophage genes, RNA general metabolism genes and other metabolism genes. We focused on mutations in lacI (lac operon repressor), iraM (anti-RssB factor, involved in RpoS stabilization during $\mathrm{Mg}$ starvation) and $m h p R$ (transcriptional regulator) genes. Modifications in these three genes were observed in the chromosome of the five strains analyzed. As shown in Fig. 3, the lacI and iraM mutations were unable to mask MqsR toxicity; however, GhoT was not toxic in both of the iraM and $m h p R$ strains, and MqsR was inactive in the $m p h R$ strain. Hence, inactivating MhpR renders both GhoT and MqsR toxins inactive.

Bioinformatic analysis. To determine the presence and conservation of these toxins in E. coli, we made a bioinformatic analysis of $1,000 \mathrm{E}$. coli genomes. This analysis revealed that Hha is the most conserved toxin among the four, being present in $98 \%$ of the population analyzed (Fig. 4). Also, GhoT was conserved since it was present in the $91 \%$ of the genomes. This analysis also shows $50 \%$ of the changes in $h h a(49 \%$ with a $99 \%$ of homology and $1 \%$ with less) and 33\% of the changes in $g h o T(30 \%$ of the strains with a $99 \%$ of homology and $3 \%$ of strains with less than $99 \%$ ), compared to $50 \%$ and $67 \%$ of the population with $100 \%$ of homology in their sequences. However, both genes were present with $100 \%$ of identity in the amino acid sequence in $99 \%$ and $98 \%$ of strains, respectively, with only $1 \%$ of the population has changes in the amino acid sequence in both cases and another $1 \%$ of the population in which the modification of the nucleotide sequence was translated with an early stop in the protein in the case of GhoT.

MqsR toxin was found in 29\% of the 1,000 genomes analyzed, of which $58 \%$ have $100 \%$ nucleotide sequence homology and a $99 \%$ homology in the other $42 \%$ of the population. However, these percentages 
of homology only implied $88 \%$ of the population has $100 \%$ of homology in its amino acid sequence, and

$7 \%$ of the population has changes in the protein sequence and 5\% had an early stop codon (Fig. 4).

RalR was the less conserved toxin since it was present in only $21 \%$ of the genomes analyzed. Only $41 \%$ of the strains had $100 \%$ homology in the nucleotide sequence compared to $36 \%$ that had $99 \%$ identity and $5 \%$ showed less than $99 \%$ homology. For the RalR sequence, only $43 \%$ of the population had $100 \%$ amino acid identity, and $31 \%$ of strains had changes in the protein sequence (some with more than one amino acid change). Furthermore, we found that $26 \%$ of the genomes had an early stop codon in their sequence.

Overall, our bioinformatic results show these four toxin genes are widely distributed and conserved in E. coli genomes. Hence, these data indicate clearly the importance of these TAs are to bacteria.

\section{DISCUSSION}

In this study, we demonstrate that bacteria mutate rapidly (in the first 12 hours of toxin production) to inactivate toxins of TAs and allow the cells to resume growth, when toxin production is not regulated. Hence our data indicate the importance of tight regulation for TA systems. The mutations that inactivate the toxins were found primarily in the -10 promoter region of the plasmids that carry the toxins or in lacI, iraM and $m h p R$. No mutations were found in the structural part of the toxin genes. These results are novel since previous work has shown that toxins are inactivated by mutations in the toxin gene itself [27-29].

The lack of changes in the toxin structural genes for all four toxins is surprising since we have shown TA systems can evolve rapidly, transforming their genes into new toxins and antitoxins via a few mutations in their genes [30]. For example, a novel toxin was created from the ghoS antitoxin gene (with only two amino acid changes), and two novel antitoxins were created from the mqs $A$ and from toxI antitoxin genes [30]. Therefore, since small changes in the sequence of either a toxin or an antitoxin gene can radically alter the TA system, perhaps this prevents mutations in the toxin structural gene.

The whole-genome sequencing revealed the two chromosomal mutations that we verified inactivate the toxins (Fig. 3). The first, iraM encodes an anti-adaptor protein that has been related to the stabilization of RpoS during Mg starvation [31-33]. IraM has also been linked to the PhoP/PhoQ a two component system, 
which is necessary to activate IraM, and to H-NS that inhibits IraM activation [34]. The second mutation, $m h p R$, encodes a DNA-binding transcriptional activator and is located upstream of the lacI reperessor [35]; MhpR is the regulator of the 3-hydroxyphenyl propionate catabolic pathway-10 [36] and is activated by the cAMP-CRP complex in the absence of glucose and in the presence of 3-hydroxyphenyl propionate [37]. How inactivating IraM and MhpR reduce toxin activity remains to be discerned.

Bacteria with high stress increase their mutation rate; for example, the mutation rate of E.coli is higher with prolonged growth arrest [38]. For antibiotic-induced resistance mutations, some studies suggest stress induces the mutations [39] while others suggest the mutations are due to selection [40]. Our data indicate that the appearance of toxin-inactivating mutations are due to selection rather than random mutation since the same promoter deletions were found repeatedly that inactivate the toxin and select faster-growing cells.

Overall, by using four toxins from four different type of TA systems, we found toxins are rapidly inactivated by changes in their promoters rather than changes in the structural genes. We also identified two proteins important for toxin activity, IraM and MhpR, and our results suggest selection is important for mutations.

\section{METHODS}

Bacterial strains and growth conditions. Escherichia coli K12 BW25113 [41] with plasmids pCA24N [42], pCA24N-mqsR [18], pCA24N-ghoT [43], pCA24N-ralR [22] and pCA24N-hha [11] was used for producing toxins and BW25113 with plasmids pCA24N-mqsRA [18], pCA24N-ghoST [43], pCA24NralRA [22], and pCA24N-hha-tomB [11] was used for producing the toxin along with its antitoxin. Moreover, we used Keio mutants for lacI, iraM, and $m h p R$ genes (27) with the pCA24N-based plasmids. All cultures were grown in lysogeny broth (LB) [44] supplemented with $30 \mu \mathrm{g} / \mathrm{mL}$ of chloramphenicol (to maintain the plasmids) at $37^{\circ} \mathrm{C}$ with shaking.

Toxin inactivation. The toxicity of each toxin was confirmed by growing each strain from an overnight culture on plates with and without $1 \mathrm{mM}$ isopropyl $\beta$-D-1-thiogalactopyranoside (IPTG). For inactivation, overnight cultures were used to inoculate fresh medium and grown until a turbidity at $600 \mathrm{~nm}$ of 0.05 to 
0.1 , then $1 \mathrm{mM}$ IPTG was added. The turbidity was measured every 15 minutes until it reached 0.7 to 0.8 . After 12 hours, single colonies were isolated from each culture, regrown, and the plasmid purified with an E.Z.N.A plasmid DNA Minikit Omega ${ }^{\circledR}$ for sequencing. To confirm that the toxins had been inactivated, the purified strains were grown in liquid cultures in the presence of $1 \mathrm{mM}$ of IPTG.

Bioinformatic analysis. Sequences were analyzed by Clustal Omega. The analysis of 1,000 genomes of $E$. coli were made using "Integrated microbial genomes and microbiomes" (IMG/M).

Mutation analysis. Plasmids were sequenced (Quintara Biosciences) using reverse primer pCA24N-R (5'GAACAAATCCAGATGGAGTTCTGAGGTCATT-3'). Strains that lost toxin activity but did not show any mutations in the plasmid-based toxin gene or its promoter were sequenced by Illumina HiSeq platform. Raw sequence data were trimmed by Sickle (https://github.com/najoshi/sickle) and quality was checked by FastQC (https://www.bioinformatics.babraham.ac.uk/projects/fastqc/). The raw data were mapped to reference the genome by BWA (http://bio-bwa.sourceforge.net/). Mapping data were sorted, merged, and deduplicated by Picard (https://broadinstitute.github.io/picard/). Realignment and unified-genotype data were performed by GATK (https://software.broadinstitute.org/gatk/). SnpEff (http://snpeff.sourceforge.net/) was used for genome annotation. REDTools (https://bedtools.readthedocs.io/en/latest/) and Samtools (http://samtools.sourceforge.net/) were used for 170 calculating the depth of each base and for finding unique reads. All the strains were re-grown in the presence of $1 \mathrm{mM}$ of IPTG after the mutation period, in order to assay the stability of the mutations, by measuring the turbidity at $600 \mathrm{~nm}$.

\section{ACKNOWLEDGEMENTS}

This work was supported in part by funds derived from the Biotechnology Endowed Professorship at 175 the Pennsylvania State University (for TKW), by a SEIMC grant and predoctoral fellowship from the Xunta de Galicia (GAIN, Axencia de Innovación) (for LFG), by the KRIBB Initiative Research Program (for JSK), and by grant PI16/01163 within the State Plan for R+D+I 2013-2016 (National Plan for Scientific Research, Technological Development and Innovation 2008-2011), by the ISCIII-Deputy General 
Directorate for Evaluation and Promotion of Research - European Regional Development Fund "A way of

Making Europe," by the Instituto de Salud Carlos III FEDER, by the Spanish Network for the Research in Infectious Diseases (REIPI, RD16/0016/0006), and by the Study Group on Mechanisms of Action and Resistance to Antimicrobials, GEMARA (for MT). The authors have no conflicts of interest.

\section{REFERENCES}

1. Wang, X.; Kim, Y.; Hong, S.H.; Ma, Q.; Brown, B.L.; Pu, M.; Tarone, A.M.; Benedik, M.J.; Peti, W.; Page, R., et al. Antitoxin MqsA helps mediate the bacterial general stress response. Nat Chem Biol 2011, 7, 359-366.

2. Kim, Y.; Wood, T.K. Toxins Hha and CspD and small RNA regulator Hfq are involved in persister cell formation through MqsR in Escherichia coli. Biochem Biophys Res Commun 2010, 391, 209-213.

3. Shah, D.; Zhang, Z.; Khodursky, A.; Kaldalu, N.; Kurg, K.; Lewis, K. Persisters: a distinct physiological state of E. coli. BMC Microbiol 2006, 6, 53.

4. Pecota, D.C.; Wood, T.K. Exclusion of T4 Phage by the hok/sok Killer Locus from Plasmid R1. J Bacteriol 1996, 178, 2044-2050.

5. Schuster, C.F.; Bertram, R. Toxin-anJtitoxin systems are ubiquitous and versatile modulators of prokaryotic cell fate. FEMS Microbiol Lett 2013, 340, 73-85.

6. Ogura, T.; Hiraga, S. Mini-F plasmid genes that couple host cell division to plasmid proliferation. PNAS 1983, 80, 4784-4788.

7. Page, R.; Peti, W. Toxin-antitoxin systems in bacterial growth arrest and persistence. Nat Chem Biol 2016, 12, 208-214.

8. Yamaguchi, Y.; Park, J.H.; Inouye, M. Toxin-antitoxin systems in bacteria and archaea. Annu Rev Genet 2011, 45, 61-79.

9. Masuda, H.; Tan, Q.; Awano, N.; Wu, K.P.; Inouye, M. YeeU enhances the bundling of cytoskeletal polymers of MreB and FtsZ, antagonizing the CbtA (YeeV) toxicity in Escherichia coli. Mol Microbiol 2012, 84, 979-989.

10. Wang, X.; Lord, D.M.; Cheng, H.-Y.; Osbourne, D.O.; Hong, S.H.; Sanchez-Torres, V.; Quiroga, C.; Zheng, K.; Herrmann, T.; Peti, W., et al. A Novel Type V TA System Where mRNA for Toxin GhoT is Cleaved by Antitoxin GhoS. Nat. Chem. Biol. 2012, 8, 855-861.

11. Marimon, O.; Teixeira, J.M.; Cordeiro, T.N.; Soo, V.W.; Wood, T.L.; Mayzel, M.; Amata, I.; García, J.; Morera, A.; Gay, M., et al. An oxygen-sensitive toxin-antitoxin system. Nat Commun 2016, 7, 13634.

12. Song, S.; Wood, T.K. Post-segregational Killing and Phage Inhibition Are Not Mediated by Cell Death Through Toxin/Antitoxin Systems. Front Microbiol 2018, 9, 814.

13. Brown, B.L.; Grigoriu, S.; Kim, Y.; Arruda, J.M.; Davenport, A.; Wood, T.K.; Peti, W.; Page, R. Three dimensional structure of the MqsR:MqsA complex: A novel toxin:antitoxin pair comprised of a toxin homologous to RelE and an antitoxin with unique properties. PLoS Pathog. 2009, 5, e1000706.

14. Hayes, F.; Kędzierska, B. Regulating toxin-antitoxin expression: controlled detonation of intracellular molecular timebombs. Toxins (Basel) 2014, 6, 337-358.

15. Wang, X.; Lord, D.M.; Hong, S.H.; Peti, W.; Benedik, M.J.; Page, R.; Wood, T.K. Type II toxin/antitoxin MqsR/MqsA controls type V toxin/antitoxin GhoT/GhoS. Environ Microbiol 2013, 15, 1734-1744.

16. Brown, B.L.; Lord, D.M.; Grigoriu, S.; Peti, W.; Page, R. The Escherichia coli toxin MqsR destabilizes the transcriptional repression complex formed between the antitoxin MqsA and the mqsRA operon promoter. J Biol Chem 2012, 288, 1286-1294. 
17. Ren, D.; Bedzyk, L.A.; Thomas, S.M.; Ye, R.W.; Wood, T.K. Gene expression in Escherichia coli biofilms. Appl Microbiol Biotechnol 2004, 64, 515-524.

18. Wang, X.; Lord, D.M.; Hong, S.H.; Peti, W.; Benedik, M.J.; Page, R.; Wood, T.K. Type II toxin/antitoxin MqsR/MqsA controls type V toxin/antitoxin GhoT/GhoS. Environ Microbiol 2013, 15, 1734-1744.

19. Kwan, B.W.; Lord, D.M.; Peti, W.; Page, R.; Benedik, M.J.; Wood, T.K. The MqsR/MqsA toxin/antitoxin system protects Escherichia coli during bile acid stress. Environ Microbiol 2015, 17, 3168-3181.

20. Kim, Y.; Wood, T.K. Toxins Hha and CspD and small RNA regulator Hfq are involved in persister cell formation through MqsR in Escherichia coli. Biochem Biophys Res Commun 2009, 391, 209-213.

21. Cheng, H.-Y.; Soo, V.W.C.; Islam, S.; McAnulty, M.J.; Benedik, M.J.; Wood, T.K. Toxin GhoT of the GhoT/GhoS toxin/antitoxin system damages the cell membrane to reduce adenosine triphosphate and to reduce growth under stress. Environmental Microbiology 2014, 16, 1741-1754.

22. Guo, Y.; Quiroga, C.; Chen, Q.; McAnulty, M.J.; Benedik, M.J.; Wood, T.K.; Wang, X. RalR (a DNase) and RalA (a small RNA) form a type I toxin-antitoxin system in Escherichia coli. Nucleic Acids Res 2014, 42, 6448-6462.

23. Hong, S.H.; Lee, J.; Wood, T.K. Engineering global regulator Hha of Escherichia coli to control biofilm dispersal. Microb Biotechnol 2010, 3, 717-728.

24. García-Contreras, R.; Zhang, X.S.; Kim, Y.; Wood, T.K. Protein translation and cell death: the role of rare tRNAs in biofilm formation and in activating dormant phage killer genes. PLoS One 2008, 3, e2394.

25. Balsalobre, C.; Johansson, J.; Uhlin, B.E.; Juárez, A.; Muñoa, F.J. Alterations in protein expression caused by the hha mutation in Escherichia coli: influence of growth medium osmolarity. $J$ Bacteriol 1999, 181, 3018-3024.

26. Wang, X.; Kim, Y.; Wood, T.K. Control and benefits of CP4-57 prophage excision in Escherichia coli biofilms. ISME J 2009, 3, 1164-1179.

27. Masachis, S.; Tourasse, N.J.; Chabas, S.; Bouchez, O.; Darfeuille, F. Chapter Four - FASTBAC-Seq: Functional Analysis of Toxin-Antitoxin Systems in Bacteria by Deep Sequencing. In Methods in Enzymology, Carpousis, A.J., Ed. Academic Press: 2018; Vol. 612, pp. 67-100.

28. Silvaggi, J.M.; Perkins, J.B.; Losick, R. Small Untranslated RNA Antitoxin in Bacillus subtilis. Journal of Bacteriology 2005, 187, 6641-6650.

29. Jahn, N.; Preis, H.; Wiedemann, C.; Brantl, S. BsrG/SR4 from Bacillus subtilis- the first temperaturedependent type I toxin-antitoxin system. Molecular Microbiology 2012, 83, 579-598.

30. Soo, V.W.; Cheng, H.Y.; Kwan, B.W.; Wood, T.K. de novo synthesis of a bacterial toxin/antitoxin system. Sci Rep 2014, 4, 4807.

31. Bougdour, A.; Cunning, C.; Baptiste, P.J.; Elliott, T.; Gottesman, S. Multiple pathways for regulation of sigmaS (RpoS) stability in Escherichia coli via the action of multiple anti-adaptors. Mol Microbiol 2008, 68, 298-313.

32. Yang, H.; Wolff, E.; Kim, M.; Diep, A.; Miller, J.H. Identification of mutator genes and mutational pathways in Escherichia coli using a multicopy cloning approach. Mol Microbiol 2004, 53, 283-295.

33. Hemmi, H.; Ohnuma, S.; Nagaoka, K.; Nishino, T. Identification of genes affecting lycopene formation in Escherichia coli transformed with carotenoid biosynthetic genes: candidates for early genes in isoprenoid biosynthesis. J Biochem 1998, 123, 1088-1096.

34. Battesti, A.; Tsegaye, Y.M.; Packer, D.G.; Majdalani, N.; Gottesman, S. H-NS regulation of IraD and IraM antiadaptors for control of RpoS degradation. J Bacteriol 2012, 194, 2470-2478.

35. Zhang, G.-q.; Cao, Z.-w.; Luo, Q.-m.; Cai, Y.-d.; Li, Y.-x. Operon prediction based on SVM. Computational Biology and Chemistry 2006, 30, 233-240.

36. Manso, I.; García, J.L.; Galán, B. Escherichia coli $\mathrm{mhpR}$ gene expression is regulated by catabolite repression mediated by the cAMP-CRP complex. Microbiology 2011, 157, 593-600.

37. Torres, B.; Porras, G.; Garcia, J.L.; Diaz, E. Regulation of the mhp cluster responsible for 3-(3hydroxyphenyl)propionic acid degradation in Escherichia coli. J Biol Chem 2003, 278, 27575-27585. 
38. Loewe, L.; Textor, V.; Scherer, S. High deleterious genomic mutation rate in stationary phase of Escherichia coli. Science 2003, 302, 1558-1560.

39. Bjedov, I.; Tenaillon, O.; Gérard, B.; Souza, V.; Denamur, E.; Radman, M.; Taddei, F.; Matic, I. Stressinduced mutagenesis in bacteria. Science 2003, 300, 1404-1409.

40. Wrande, M.; Roth, J.R.; Hughes, D. Accumulation of mutants in "aging" bacterial colonies is due to growth under selection, not stress-induced mutagenesis. Proc Natl Acad Sci U S A 2008, 105, 11863 11868.

41. Baba, T.; Ara, T.; Hasegawa, M.; Takai, Y.; Okumura, Y.; Baba, M.; Datsenko, K.A.; Tomita, M.; Wanner, B.L.; Mori, H. Construction of Escherichia coli K-12 in-frame, single-gene knockout mutants: the Keio collection. Mol Syst Biol 2006, 2, 20060008.

42. Kitagawa, M.; Ara, T.; Arifuzzaman, M.; Ioka-Nakamichi, T.; Inamoto, E.; Toyonaga, H.; Mori, H. Complete set of ORF clones of Escherichia coli ASKA library (a complete set of E. coli K-12 ORF archive): unique resources for biological research. DNA Res 2005, 12, 291-299.

43. Wang, X.; Lord, D.M.; Cheng, H.Y.; Osbourne, D.O.; Hong, S.H.; Sanchez-Torres, V.; Quiroga, C.; Zheng, K.; Herrmann, T.; Peti, W., et al. A new type V toxin-antitoxin system where mRNA for toxin GhoT is cleaved by antitoxin GhoS. Nat Chem Biol 2012, 8, 855-861.

44. Sambrook, J.; Fritsch, E.F.; Maniatis, T. Molecular cloning : a laboratory manual, 2nd ed.; pp. 3 volumes. 


\section{FIGURE CAPTIONS}

Fig. 1. Inactivation of plasmid-based toxins. Plate results: BW25113 producing toxins from pCA24Nbased plasmids (pCA24N-mqsR, pCA24N-ghoT, pCA24N-ralR, or pCA24N-hha) with (left) and without (right) $1 \mathrm{mM}$ IPTG to induce the toxin genes indicate the initial toxicity for MqsR, GhoT, RalR, and Hha. Five colonies of the original strain were streaked on each plate. Growth curves: Growth of cells harboring the empty plasmid pCA24N (“Empty”, black circles and lines), the toxin and antitoxin (blue diamonds and lines), and toxin alone (triangles and red lines) with 1mM IPTG.

Fig. 2. Stability of the toxin inactivation mutations. Growth of BW25113 containing pCA24N-based plasmids with mutated promoters or chromosomal changes that inactivate the toxin in the presence of $1 \mathrm{mM}$ IPTG. Blue indicates plasmid mutations whereas red indicates chromosomal changes. Sequences of the plasmid toxin genes and promoter are shown in Fig. S1 (for mqsR), Fig. S2 (for ghoT), Fig. S3 (for ralR), and Fig. S4 (for hha).

Fig. 3. Inactivation of plasmid-based toxins by chromosomal mutations. Growth of the (a) lacI, (b) iraM, and (c) $m h p R$ strains containing the empty plasmid pCA24N (black), pCA24N-mqsR (blue), or pCA24N-ghoT (green) in the presence of $1 \mathrm{mM}$ IPTG to induce the toxins.

Fig. 4. Conservation of the toxins in $E$. coli. Presence (\%, first column), nucleotide identity (\%, $2^{\text {nd }}$ column) and amino acid identity $\left(\%, 3^{\text {rd }}\right.$ column) of each toxin in 1,000 E. coli genomes. 
a) MqsR/MqsA system

a.1)

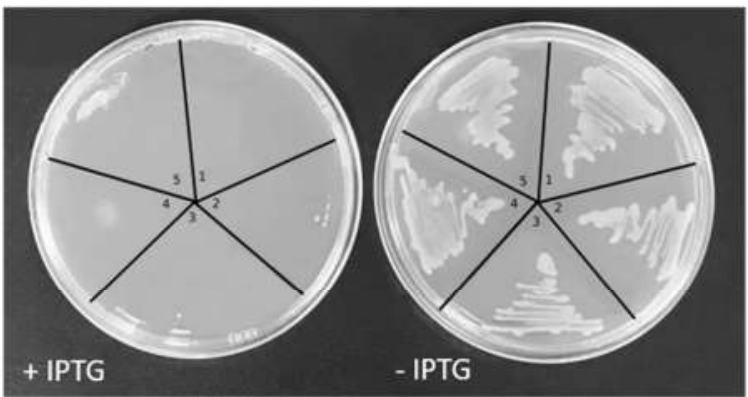

b) GhoT/GhoS system

b.1)

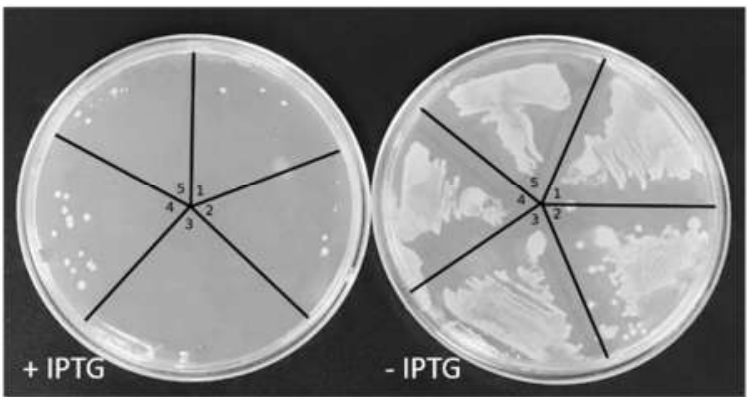

c) RaIR/ RalA system

c.1)

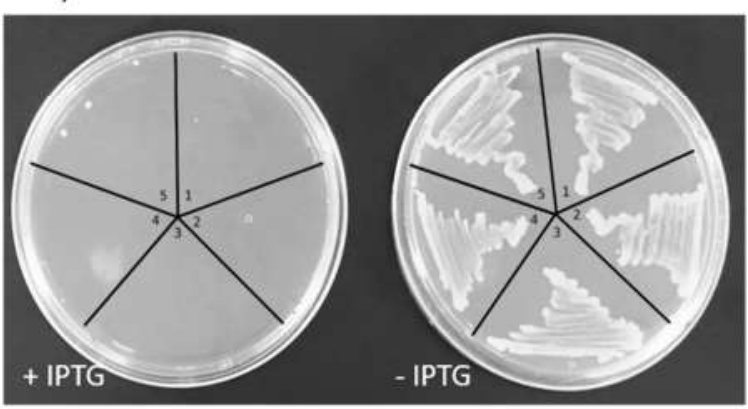

d) Hha/Tom system

d.1)

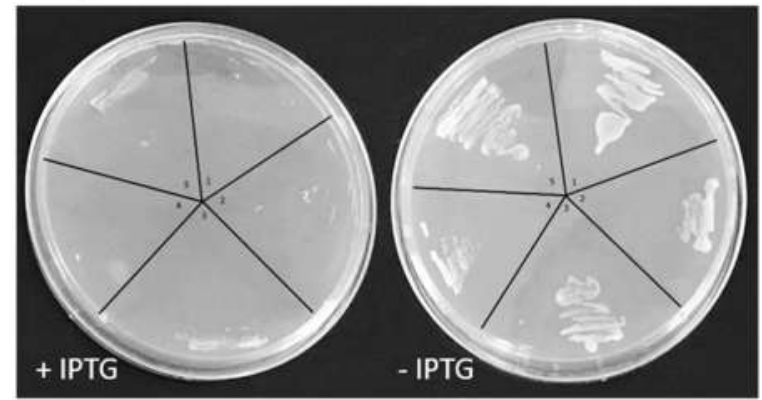

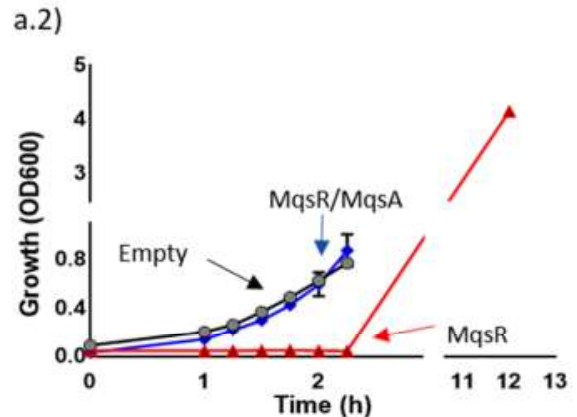
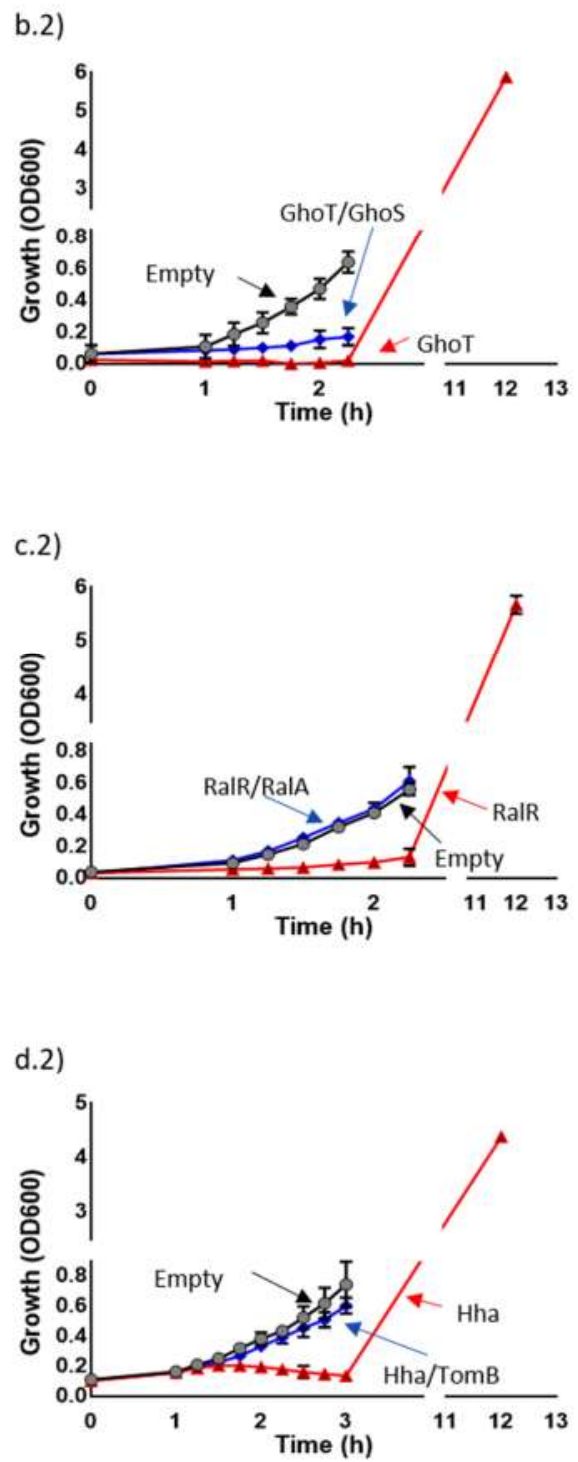

Figure 1 
a) MqsR

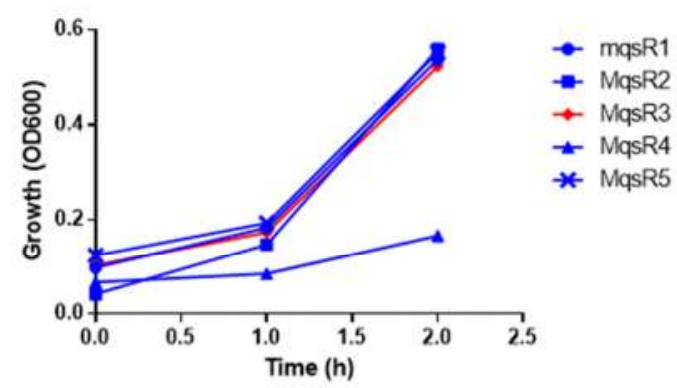

c) RalR

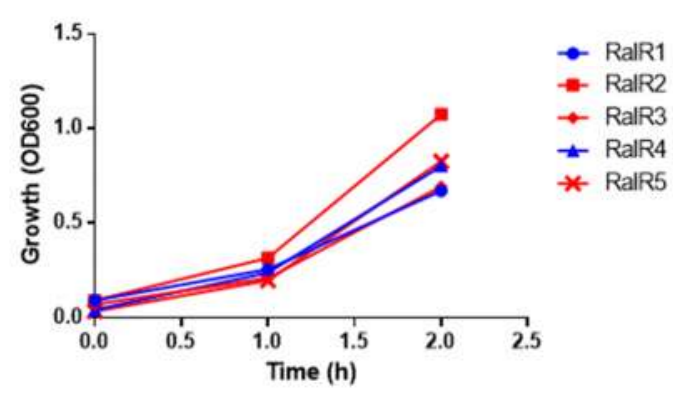

b) GhoT

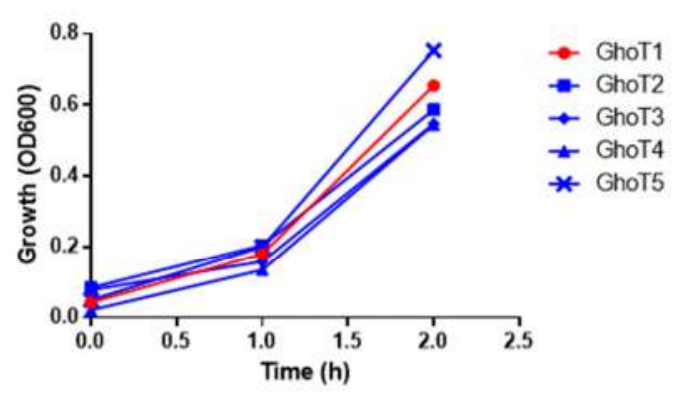

d) Hha

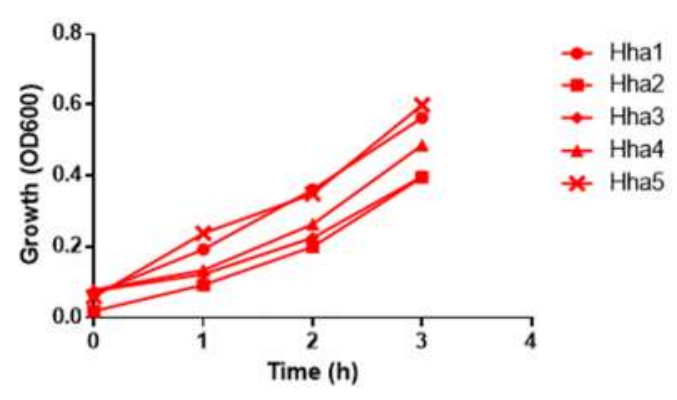

Figure 2 
a) $\Delta \mathrm{lacl}$

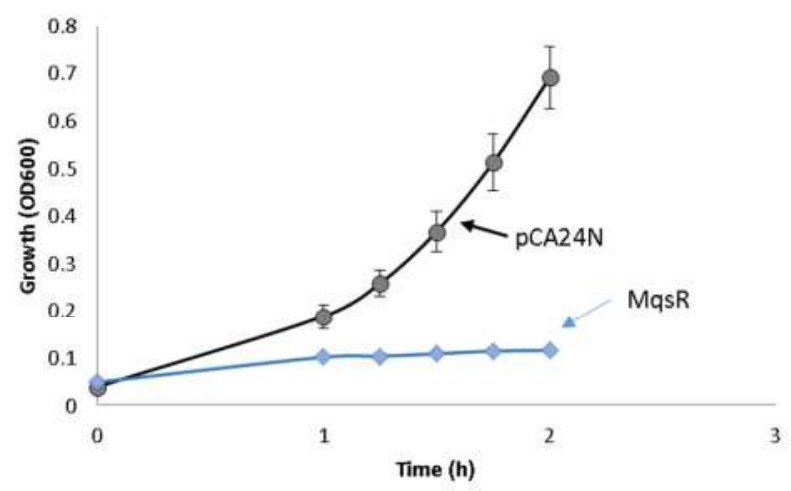

C) $\triangle m h p R$

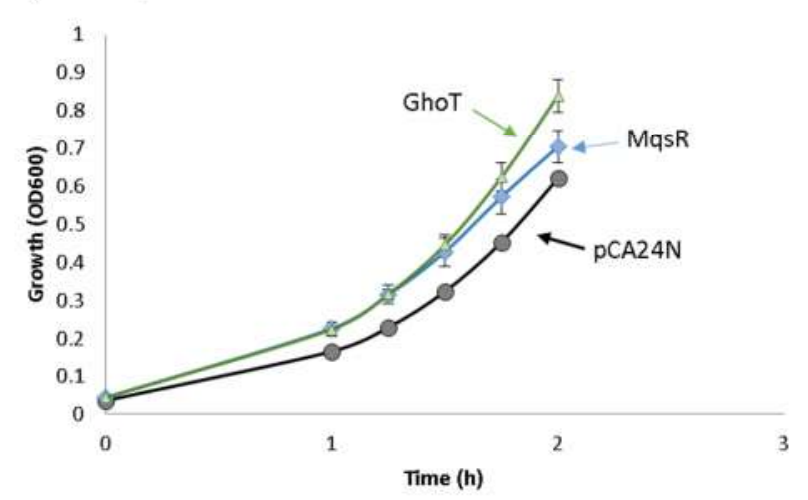

b) $\Delta i r a M$

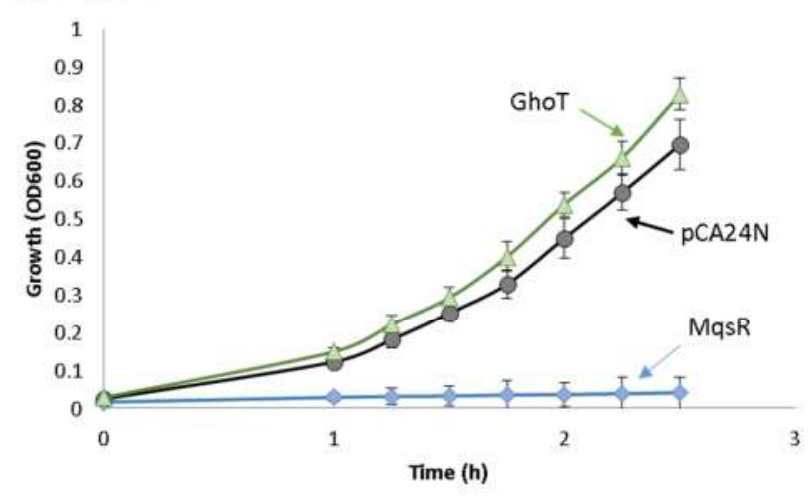

\section{Figure 3}




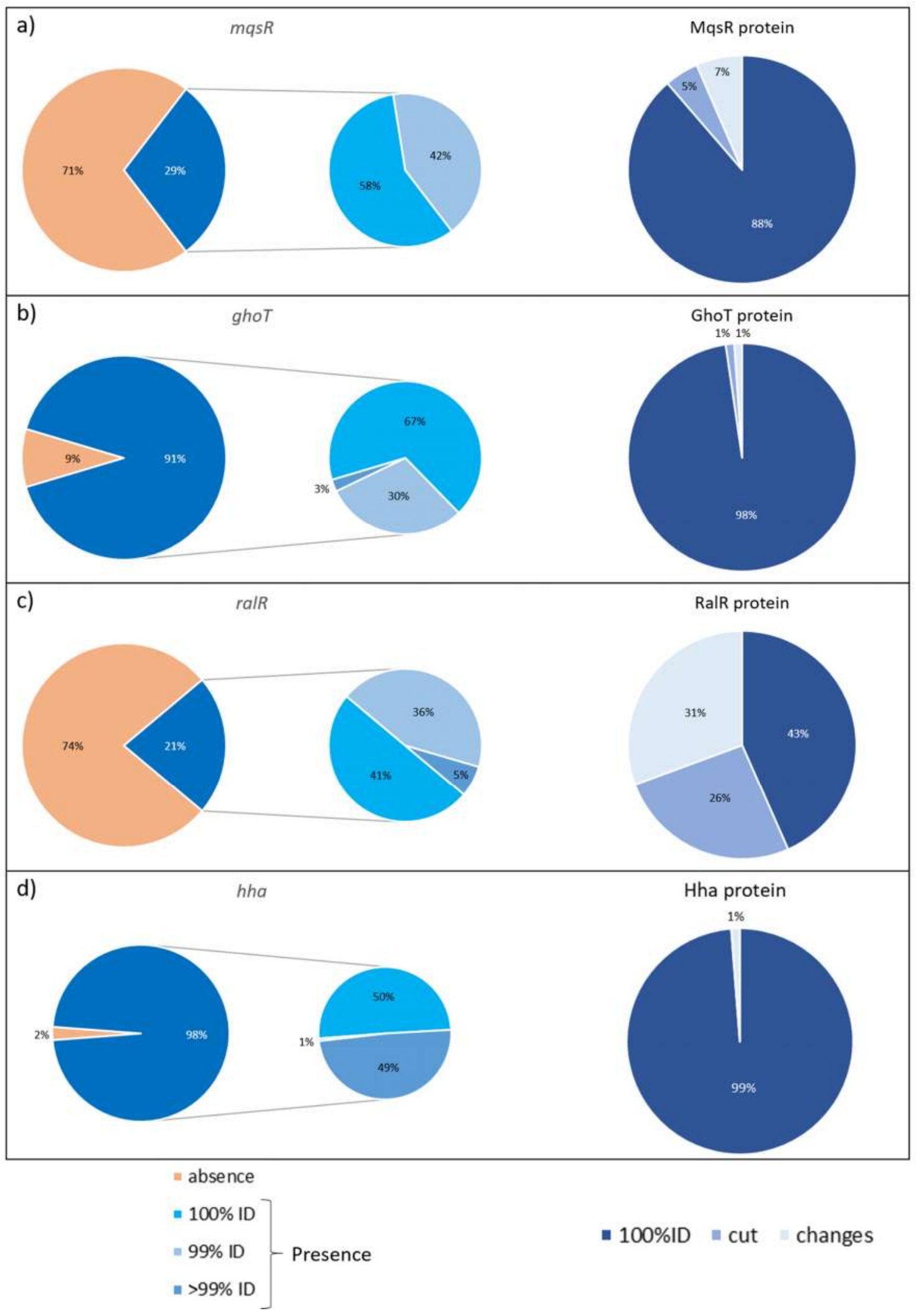

Figure 4

Fernández García et. al. 


\section{SUPPLEMENTARY INFORMATION}

\section{Toxin Inactivation in Toxin/Antitoxin Systems}

\section{Laura Fernandez-Garica ${ }^{1,3}$, Jun-Seob Kim ${ }^{4}$, Maria Tomas ${ }^{3}$, and Thomas K. Wood ${ }^{1,2, *}$}

${ }^{1}$ Department of Chemical Engineering and ${ }^{2}$ Department of Biochemistry and Molecular Biology, Pennsylvania State University, University Park, Pennsylvania, 16802-4400, USA ${ }^{3}$ Microbiology Department-Research Institute Biomedical A Coruña (INIBIC), Hospital A Coruña (CHUAC), University of A Coruña (UDC), A Coruña, Spain

${ }^{4}$ Infectious Disease Research Center, Korea Research Institute of Bioscience \& Biotechnology (KRIBB) 125 Gwahak-ro, Yuseong-gu, Daejeon 34141, South Korea

*For correspondence. E-mail tuw14@psu.edu

Tel.(+)1 814-863-4811; Fax (1) 814-865-7846 


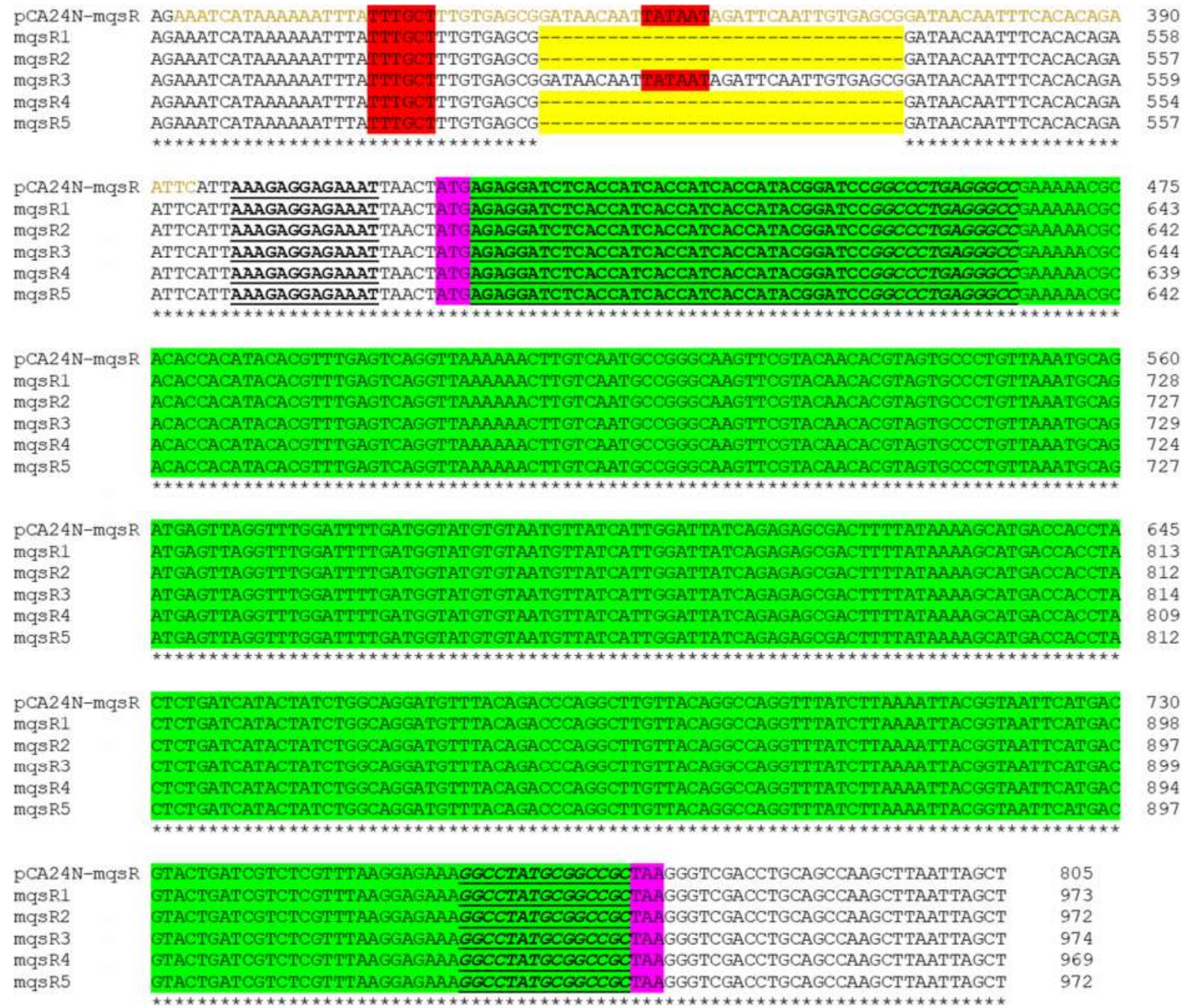

Fig. S1. Plasmid sequences for inactivated MqsR. Red highlight indicates the -10 and -35 promoter regions, yellow highlight indicates promoter deletions, yellow letters indicate the pt5-lac promoter, bold and underlined letters indicate the RBS, green highlight indicates the coding portion of the gene sequence, bold green highlight at the 5' end contains the 6x His tag, italic bold green highlight at the 5' and 3'ends indicates SfiI restriction sites, and pink highlight indicates the start and stop codons. 


\begin{tabular}{|c|c|}
\hline pCA2 $4 \mathrm{~N}-\mathrm{ghoT}$ & 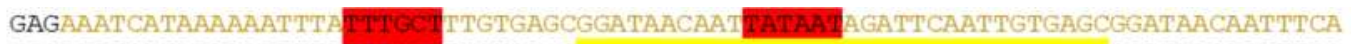 \\
\hline ghoT2 & CGGAAATCATAAAAAATTTA TITGCTTTGTGAGC- \\
\hline ghoT1 & GAGAAATCATAAAAAATTTA TTECNTGTGAGCGGATAACAATTATAATAGATTCAATTGTGAGCGGATAACAATTTCA \\
\hline ghot4 & CGGAAATCATAAAAAATTTA TTTGMTTTGAGC- \\
\hline ghot3 & 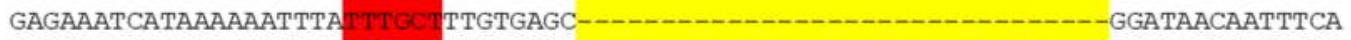 \\
\hline ghoT5 & 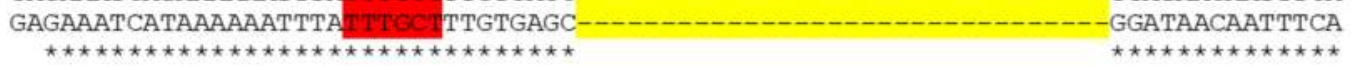 \\
\hline pCA2 $4 \mathrm{~N}-\mathrm{ghoT}$ & CACAGAATT CATTAAAGAGGAGAAATTAACT ATGAGAGGATCTCACCATCACCATCACCATACGGATCCGGCCCTGAGGG \\
\hline ghoT2 & CACAGAATTCATTAAAGAGGAGAAATTAACT ATGAGAGGATCTCACCATCACCATCACCATACGGATCCGGCCCTGAGGG \\
\hline ghoT1 & CACAGAATTCATTAAAGAGGAGAAATTAACT ATGAGAGGATCTCACCATCACCATCACCATACGGATCCGGCCCTGAGGG \\
\hline ghoT4 & CACAGAATTCATTAAAGAGGAGAAATTAACT AT GAGAGGATCTCACCATCACCATCACCATACGGATCCGGCCCTGAGGG \\
\hline ghot3 & CACAGAATTCATTAAAGAGGAGAAATTAACT AT GAGAGGATCTCACCATCACCATCACCATACGGATCCGGCCCTGAGGG \\
\hline & 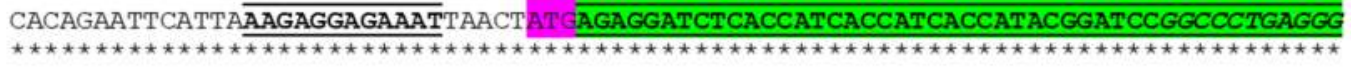 \\
\hline pCA24N-ghoT & COGCACTATTCTCTAAAATATTAATTTTTATGTGATTGGTGTGAACATATCCTTTGTCATTATCTGGTTATCTCACAT \\
\hline ghoT2 & COGCACTATTCTCTAAAATATTAATTTTTTATGTGATTGGTGTGAACATATCCTTTGTCATTATCTGGTTTATCTCACAT \\
\hline ghot1 & COGCACTATTCTCTAAAATATTAATTTTTATGTGATTGGTGTGAACATATCCTTTGTCATTATCTGGTTTATCTCACAT \\
\hline ghot4 & COGCACTATTCTCTAAAATATTAATTTTTATGTGATTGGTGTGAACATATCCTTTGTCATTATCTGGTTATCTCACAT \\
\hline ghot3 & $\overline{C O}$ GCACTATTCTCTAAAATATTAATTTTTATGTGATTGGTGTGAACATATCCTTTGTCATTATCTGGTTTATCTCACAT \\
\hline & COGGACTATTCTCTAAAATATTAATTTTTATGTGATTGGTGTGAACATATCCTTTGTCATTATCTGGTTTATCTCACAT \\
\hline pCA24N-ghoT & GAGAAAACACATATTCGTTTACTTAGTGCATTCCTGGTCGGAATAACCTGGCCAATGAGTCTGCCTGTGGCATTACTTTT \\
\hline ghoT2 & GAGAAAACACATATTCGTTTACTTAGTGCATTCCTGGTCGGAATAACCTGGCCAATGAGTCTGCCTGTGGCATTACTTTT \\
\hline ghoT1 & GAGAAAACACATATTCGTTTACTTAGTGCATTCCTGGTCGGAATAACCTGGCCAATGAGTCTGCCTGTGGCATTACTTTT \\
\hline ghoT4 & GAGAAAACACATATTCGTTTACTTAGTGCATTCCTGGTCGGAATAACCTGGCCAATGAGTCTGCCTGTGGCATTACTTTT \\
\hline ghot3 & GAGAAAACACATATTCGTTTACTTAGTGCATTCCTGGTCGGAATAACCTGGCCAATGAGTCTGCCTGTGGCATTACTTTT \\
\hline ghot5 & GAGAAAACACATATTCGTTTACTTAGTGCATTCCTGGTCGGAATAACCTGGCCAATGAGTCTGCCTGTGGCATTACTTTT \\
\hline & ॠ \\
\hline pCA24N-ghoT & TTCTCTCTTTGGCCTATGCGGCCGCIAAGGGTCGACCTGCAGCCAAGCTTAATTAGCTGA \\
\hline ghoT2 & TTCTCTCTTT GGCCTATGCGGCCGCTAAAGGTCGACCTGCAGCCAAGCTTAATTAGCTGA \\
\hline ghoT1 & TTCTCTCTTT GGCCTATGCGGCCGCIAAGGGTCGACCTGCAGCCAAGCTTAATTAGCTGA \\
\hline ghot4 & TTCTCTCTTT GGCCTATGCGGCCGCTAAGGGTCGACCTGCAGCCAAGCTTAATTAGCTGA \\
\hline ghot3 & TTCTCTCTTT GGCCTATGCGGCCGCTAAGGGTCGACCTGCAGCCAAGCTTAATTAGCTGA \\
\hline ghoT5 & TTCTCTCTTT GGCCTATGCGGCCGCTAAGGGTCGACCTGCAGCCAAGCTTAATTAGCTGA \\
\hline
\end{tabular}

Fig. S2. Plasmid sequences for inactivated GhoT. Red highlight indicates the -10 and -35 promoter regions, yellow highlight indicates promoter deletions, yellow letters indicate the pt5-lac promoter, bold and underlined letters indicate the RBS, green highlight indicates the coding portion of the gene sequence, bold green highlight at the 5' end contains the 6x His tag, italic bold green highlight at the 5' and 3'ends indicates SfiI restriction sites, and pink highlight indicates the start and stop codons. 


pCA24N-ralR
ralR1
ralR2
ralR3
ralR4
ralR5

pCA24N-ralR
ralR1
ralR2
ralR3
ralR4
ralR5

pCA24N-ralR
ralR1
ralR2
ralR3
ralR4
ralR5

pCA24N-ralR
ralR1
ralR2
ralR3
ralR4
ralR5
pCA24N-ralR
ralR1
ralR2
ralR3
ralR4
ralR5

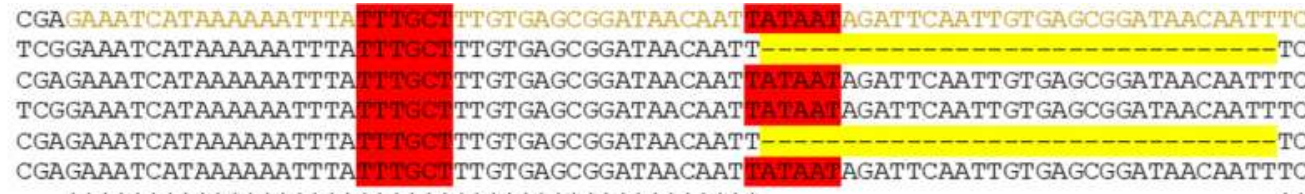
CGAGAAATCATAAAAAATTTA

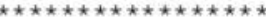
$\star \star \star \star * \star *$

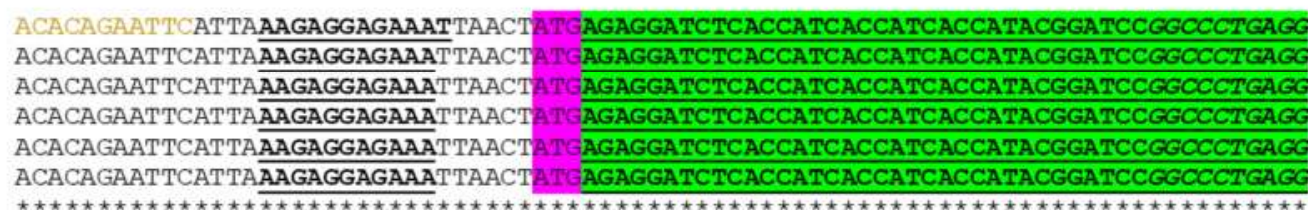
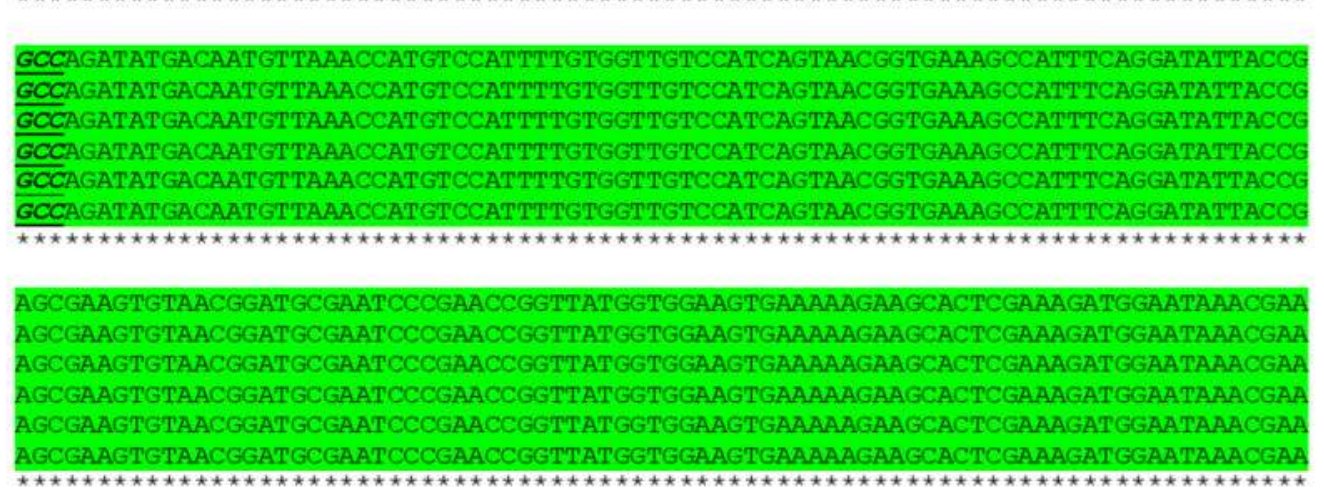
GGGTCGACCTGCAGCCAAGCTTAATTAGCT GGGTCGACCTGCAGCCAAGCTTAATTAGCT GGGTCGACCTGCAGCCAAGCTTAATTAGCT GGGTCGACCTGCAGCCAAGCTTAATTAGCT

Fig. S3. Plasmid sequences for inactivated RalR. Red highlight indicates the -10 and -35 promoter regions, yellow highlight indicates promoter deletions, yellow letters indicate the pt5-lac promoter, bold and underlined letters indicate the RBS, green highlight indicates the coding portion of the gene sequence, bold green highlight at the 5' end contains the $6 \mathrm{x}$ His tag, italic bold green highlight at the 5' and 3'ends indicates SfiI restriction sites, and pink highlight indicates the start and stop codons. 


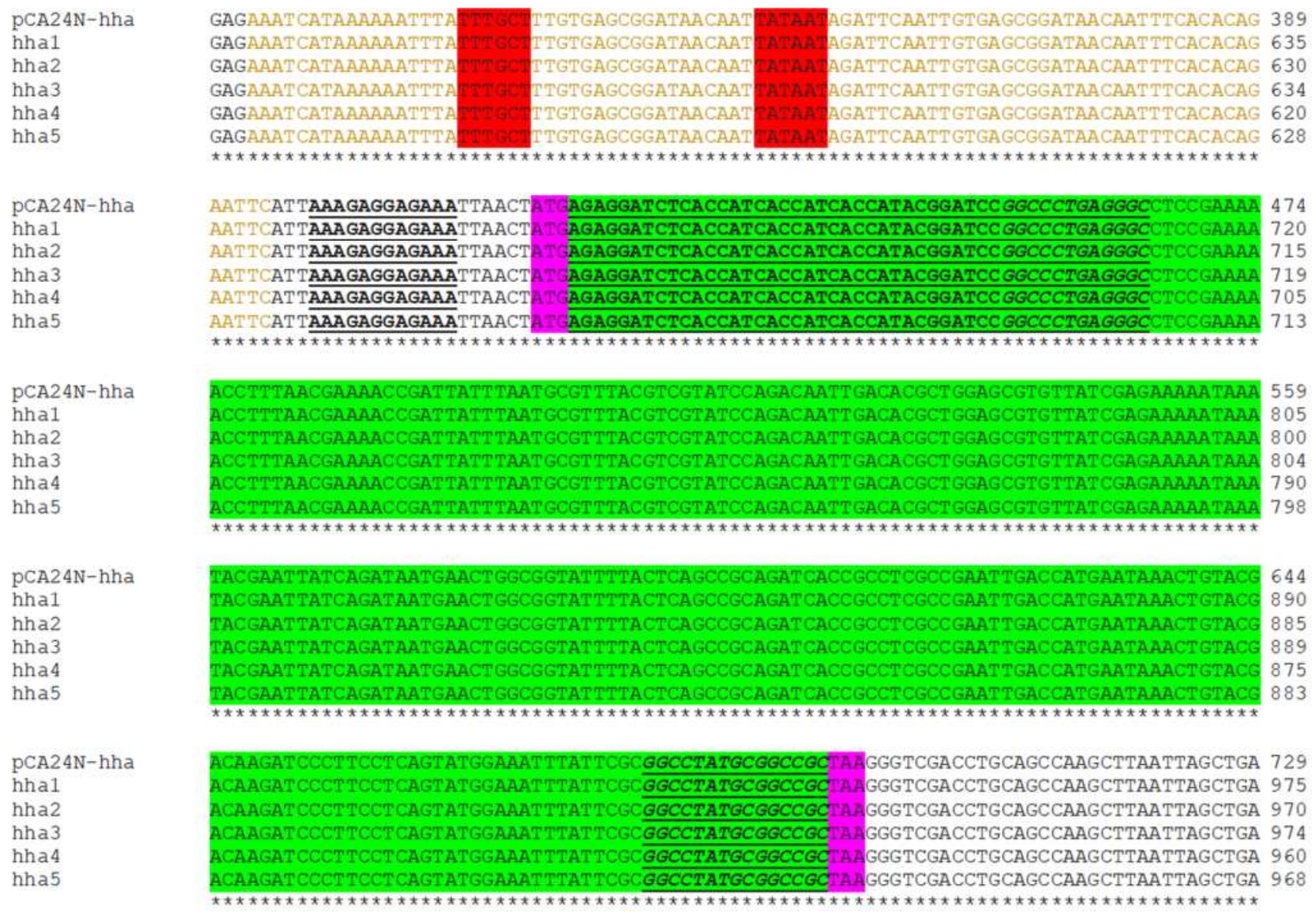

Fig. S4. Plasmid sequences for inactivated Hha. Red highlight indicates the -10 and -35 promoter regions, yellow highlight indicates promoter deletions, yellow letters indicate the pt5-lac promoter, bold and underlined letters indicate the RBS, green highlight indicates the coding portion of the gene sequence, bold green highlight at the 5' end contains the 6x His tag, italic bold green highlight at the 5' and 3'ends indicates SfiI restriction sites, and pink highlight indicates the start and stop codons. 